\title{
Is the accelerated expansion evidence of a forthcoming change of signature on the brane?
}

\author{
Marc Mars \\ Dept. de Física Fundamental, Universidad de Salamanca, \\ Plaza de la Merced s/n, 37008 Salamanca, Spain \\ José M. M. Senovilla and Raül Vera \\ Física Teórica, Universidad del País Vasco, Apartado 644, 48080 Bilbao, Spain
}

\begin{abstract}
We show that regular changes of signature on brane-worlds in $\mathrm{AdS}_{5}$ bulks may account for some types of the recently fashionable sudden singularities. Therefore, the possibility that the Universe seems to approach a future sudden singularity at an accelerated rate of expansion might simply be an indication that our braneworld is about to change from Lorentzian to Euclidean signature. Both the brane and the bulk remain fully regular everywhere. We present a model in which the weak and strong energy conditions hold on the brane, in contrast with the standard cosmologies leading to the analogous kinematical behaviour (that is, with a diverging Hubble factor).

PACS numbers: 04.50.-h, 11.25.-w, 11.27.+d, 95.36.+x, 98.80.Bp
\end{abstract}

Recently 1, 2, 3], the idea that brane-world models [4, 5] are a natural scenario for the regular description of a change of the spacetime signature has been advocated. One of the interesting, and intriguing, properties of these signature-changing branes is that, even though the change of signature may be conceived as a dramatical event within the brane, both the bulk and the brane can be fully smooth. In particular, observers living in the brane but assuming that their Universe is Lorentzian everywhere may be misled to interpret that a curvature singularity arises precisely at the signature change [1, 3].

In this note we show that a correct description of this misinterpretation might explain an accelerated expansion of the Universe, while keeping the energy density and the rest of physical variables regular and non-negative everywhere; in particular without violating the weak or strong energy conditions. Explicit models are built where the Hubble parameter certainly increases and eventually diverges (for pure Lorentzian branes with finite Hubble factor see [ $[$ ] $)$, but such that this corresponds to a pathology of the proper time, which is about to disappear mutating into a spacelike coordinate at the change of signature. The resulting accelerated expansion epoch does not need of dark, phantom, or any other exotic energy.

For our purposes here we only need to consider anti de Sitter $\left(\mathrm{AdS}_{5}\right)$ bulks. Suitable coordinate systems allow us to write the bulk line-element as

$$
d s^{2}=-\left(k+\lambda^{2} r^{2}\right) d t^{2}+\left(k+\lambda^{2} r^{2}\right)^{-1} d r^{2}+r^{2} d \Omega_{k}^{2},
$$

where $\lambda$ is a positive constant related to the negative cosmological constant of the spacetime by $\Lambda_{5}=-6 \lambda^{2}$, and $d \Omega_{k}^{2}$ is the 3 -dimensional metric of constant sectional curvature $k=1,0,-1$. When $k=0,1$ the ranges of the non-angular coordinates are $-\infty<t<\infty$ and $r>0$, while for $k=-1$ we have $r>1 / \lambda$.

Due to Corollary 2 in [3], branes with a change of signature require an asymmetric set-up, so that we need to glue a region of $\mathrm{AdS}_{5}$ with a region of another anti-de Sitter space $\widetilde{\operatorname{AdS}}_{5}$ - with a different $\tilde{\Lambda}_{5}$. All quantities referring to $\widetilde{\mathrm{AdS}}_{5}$ will carry an overtilde. The gluing is performed across appropriate hypersurfaces of $\mathrm{AdS}_{5}$ and $\widetilde{\mathrm{AdS}}_{5}$ which are mutually identified, thereby producing the brane/shell $\Sigma$.

For simplicity we will only consider branes $\Sigma$ with spherical, plane or hyperboloidal symmetry, i.e. a symmetry-preserving matching [7]. Then, ignoring the angular coordinates, the corresponding hypersurfaces are given in parametric form by $\Sigma:\{t=t(\xi), r=r(\xi)\}$ and $\tilde{\Sigma}:\{\tilde{t}=\tilde{t}(\xi), \tilde{r}=\tilde{r}(\xi)\}$. The matching implies [3] that the first fundamental form on the brane reads

$$
\left.d s^{2}\right|_{\Sigma}=N(\xi) d \xi^{2}+a^{2}(\xi) d \Omega_{k}^{2}
$$

where $a(\xi)$ is defined by $r(\xi)=\tilde{r}(\xi) \equiv a(\xi)$ and $N(\xi)$ controls the embedding functions $t(\xi)$ and $\tilde{t}(\xi)$ via

$$
\dot{t}=\frac{\sigma a}{k+\lambda^{2} a^{2}} \sqrt{\frac{\dot{a}^{2}}{a^{2}}-N\left(\frac{k}{a^{2}}+\lambda^{2}\right)}
$$

and a similar equation for $\tilde{t}$ in terms of $\tilde{\lambda}$, where $\sigma$ (and $\tilde{\sigma})$ are two signs and the dot stands for $d / d \xi . N(\xi)$ and $a(\xi)$ are arbitrary functions only restricted to satisfy that both square roots, in (2) and its tilded version, are real.

From (1), the brane $\Sigma$ changes signature if $N$ changes sign. The changes of signature happen at "instants" $\xi=$ $\xi_{s}$ where $N$ becomes, or stops being, zero. The set $S=$ $\left\{\xi_{s}\right\}$ of all such points is called the signature-changing set of $\Sigma$. In general, the brane has a Lorentzian phase $\Sigma_{L}$ where $N<0$, an Euclidean phase $\Sigma_{E}$ defined by $N>0$, and a null phase $\Sigma_{0}$ where $N=0$.

The Lorentzian part $\Sigma_{L}$ describes a Robertson-Walker spacetime with $\xi$ related to the standard cosmic time $T(\xi)$ by

$$
\dot{T}=\sqrt{-N} \text { on } \Sigma_{L}
$$


From the point of view of this Lorentzian phase, the Lorentzian geometry becomes singular at $S \cap \overline{\Sigma_{L}}$. We want to describe the type of singularity that any observers living on $\Sigma_{L}$ will believe to see there. Before that, however, let us remark that this 'singularity' concerns only the "Lorentzianity" of the brane, and can only be conceived from the inner point of view of the Lorentzian phase $\Sigma_{L}$. Both the bulks and the brane $\Sigma$ are totally regular everywhere for regular functions $N(\xi)$ and $a(\xi)>0$.

In order to ascertain how the scientists living within $\Sigma_{L}$ interpret the 'singularity' at $S \cap \overline{\Sigma_{L}}$, one first needs to compute the energy-momentum tensor $\tau_{\mu \nu}$ on the brane. To this end one must use the appropriate generalized Israel formula, which was presented in [8] and applied in [3] for the case under consideration. $\tau_{\mu \nu}$ has a simple eigenvalue $\hat{\varrho}$, a triple one $\hat{p}$ associated to $d \Omega_{k}^{2}$, and the fifth vanishes. $\varrho$ reads explicitly [1, $\underline{3}$ ]

$$
\begin{aligned}
& \frac{\kappa_{5}^{2}}{3} \epsilon_{1} \sigma \hat{\varrho}\left(2 \frac{\dot{a}^{2}}{k+a^{2} \lambda^{2}}-N\right)= \\
& \sqrt{\frac{\dot{a}^{2}}{a^{2}}-N\left(\frac{k}{a^{2}}+\tilde{\lambda}^{2}\right)}-\sqrt{\frac{\dot{a}^{2}}{a^{2}}-N\left(\frac{k}{a^{2}}+\lambda^{2}\right)},
\end{aligned}
$$

where $\kappa_{5}^{2}$ is the 5 -dimensional gravitational coupling constant and $\epsilon_{1}$ is a sign selecting which region bounded by $\Sigma$ in $\mathrm{AdS}_{5}$ is to be matched with which region bounded by $\tilde{\Sigma}$ in $\widetilde{\operatorname{AdS}}_{5}$, see $[3,9]$. $\hat{\varrho}$ and $\hat{p}$ are related by

$$
\dot{\hat{\varrho}}+\frac{d}{d \xi}\left(\log \frac{\left|2 \frac{\dot{a}^{2}}{k+a^{2} \lambda^{2}}-N\right|}{\sqrt{|N|}}\right) \varrho \hat{\varrho}+3 \frac{\dot{a}}{a}(\hat{\varrho}+\hat{p})=0,
$$

which has the interpretation of a continuity equation. For signature-changing branes, $\tau_{\mu \nu}$ is affected [1, 3] by a normalisation freedom related to the choice of volume element on the brane. Irrespective of this, one can check that $\tau_{\mu \nu}, \hat{\varrho}$ and $\hat{p}$ are regular everywhere for regular $\Sigma$. No singularitities arise at the signature change or elsewhere.

The question arises of how the observers living within the Lorentzian phase of the brane may interpret these facts, and the observations which they perform, if they believe (erroneously) that their universe is Lorentzian everywhere. It turns out that there are two different possibilities according to their level of misinformation.

The first possibility arises if the scientists living in $\Sigma_{L}$ know that the bulk universe is 5-dimensional and they live on a 4-dimensional braneworld, but they do not consider signature changes as feasible. They will assume $N<0$ everywhere, and choose the cosmic time $T$ of (3) to describe the age of the universe, hence from (1)

$$
\left.d s^{2}\right|_{\Sigma_{L}}=-d T^{2}+a^{2} d \Omega_{k}^{2} .
$$

They will also naturally normalize $\hat{\varrho}$ and $\hat{p}$ according to

$$
\varrho \equiv \hat{\varrho} \frac{\left|2 \frac{\dot{a}^{2}}{k+a^{2} \lambda^{2}}-N\right|}{\sqrt{|N|}}, p \equiv \hat{p} \frac{\left|2 \frac{\dot{a}^{2}}{k+a^{2} \lambda^{2}}-N\right|}{\sqrt{|N|}},
$$

so that the conservation law (5) adopts its standard form

$$
\dot{\varrho}+3 \frac{\dot{a}}{a}(\varrho+p)=0 .
$$

This normalization corresponds to the canonical Robertson-Walker volume element. Therefore, $\varrho$ and $p$ are the energy density and pressure measured within $\Sigma_{L}$.

However, things behave quite differently for signaturechanging branes in comparison with purely Lorentzian ones regarding the 'end of time' ('beginning' for signature changes in the past). In signature-changing branes these finales can have a non-vanishing $a$, with finite $\dot{a}$ and $\ddot{a}$, but where $a^{\prime}=d a / d T$ diverges. Indeed, $a^{\prime}=\dot{a} / \sqrt{-N}$ diverges necessarily when approaching the signature-changing set $S \cap \overline{\Sigma_{L}}$ given that $\left.\dot{a}\right|_{S} \neq 0$ (otherwise, since $N=0$ on $S$, (2) would imply $\left.\dot{t}\right|_{S}=\left.\dot{\tilde{t}}\right|_{S}=0$, which is impossible [3]). Thence, the Hubble function $H \equiv a^{\prime} / a$ diverges necessarily at $S \cap \overline{\Sigma_{L}}$. This behaviour cannot be found in pure Lorentzian brane cosmologies.

Observe that (7) may seem to imply that $\varrho$ and $p$ diverge when approaching $S \cap \overline{\Sigma_{L}}$, where $N \rightarrow 0$. Actually, this is not the case because one can prove that $\varrho$ vanishes at the signature change, and that $p$ can also be regular there in many cases [3]. To see this, let us rewrite (4) in terms of the physical quantity $\varrho$

$$
\frac{\kappa_{5}^{2}}{3} \varrho=\sigma \epsilon_{1}\left(\sqrt{\frac{a^{\prime 2}+k}{a^{2}}+\tilde{\lambda}^{2}}-\sqrt{\frac{a^{\prime 2}+k}{a^{2}}+\lambda^{2}}\right),
$$

which, we remark, holds only on $\Sigma_{L}$. From (9) it is easy to find [3] the following bounds for the energy density: $\frac{\kappa_{5}^{2}}{3}|\varrho| \leq \sqrt{\left|\tilde{\lambda}^{2}-\lambda^{2}\right|}$. This inequality is strict for $k=1$, while for $k=0$ we have the stronger $\frac{\kappa_{5}^{2}}{3}|\varrho| \leq|\tilde{\lambda}-\lambda|$. Now, (9) implies also that $\varrho$ vanishes at the change of signature,

$$
\lim _{x \rightarrow S \cap \overline{\Sigma_{L}}} \varrho=\lim _{x \rightarrow S \cap \overline{\Sigma_{L}}} \frac{3 \sigma \epsilon_{1}}{2 \kappa_{5}^{2}} \frac{\tilde{\lambda}^{2}-\lambda^{2}}{|H|}=0 .
$$

This limit together with (8) leads to

$$
\lim _{x \rightarrow S \cap \overline{\Sigma_{L}}} p=\lim _{x \rightarrow S \cap \overline{\Sigma_{L}}} \frac{\sigma \epsilon_{1}}{4 \kappa_{5}^{2}}\left(\tilde{\lambda}^{2}-\lambda^{2}\right) \frac{a^{2}}{\dot{a}|\dot{a}|} \frac{\dot{N}}{\sqrt{-N}}
$$

which shows that $p$ can be regular if $\left.\dot{N}\right|_{S}=0$. Therefore, by choosing appropriately the hypersurfaces in $\mathrm{AdS}_{5}$ and $\widehat{\mathrm{AdS}}_{5}$ one can construct signature-changing branes with both $\varrho$ and $p$ finite and well-behaved everywhere on $\overline{\Sigma_{L}}$. As a matter of fact, signature-changing branes with $\tau_{\mu \nu}$ satisfying some desirable energy conditions can be built, as we will show below.

Going to a more severe level of misinformation of the scientists living within $\Sigma_{L}$, assume not only that they are uninformed about the possibility of signature changes, but also that they even ignore they live in a brane of a 
5 -dimensional bulk. They will again assume that $N<0$ holds everywhere, and choose the cosmic time $T$ to describe the age of the universe so that the line-element is given by (6). In addition, they will also use their favourite gravitational theory to describe the Universe and to compute its energy-momentum content. This will generally lead to an 'energy-momentum tensor' which has nothing to do with the genuine $\tau_{\mu \nu}$ on the brane.

If they consider General Relativity (GR) as the correct gravitational theory, they will compute the eigenvalues of the Einstein tensor of the line-element (6) on $\Sigma_{L}$

$$
\begin{aligned}
& 8 \pi G \varrho^{(G R)}+\Lambda_{4}=\frac{3}{a^{2}}\left(a^{\prime 2}+k\right), \\
& 8 \pi G p^{(G R)}-\Lambda_{4}=-2 \frac{a^{\prime \prime}}{a}-\frac{1}{a^{2}}\left(a^{\prime 2}+k\right),
\end{aligned}
$$

which obviously diverge at the 'singularity' placed on the signature-changing set $S \cap \overline{\Sigma_{L}}$. Here, $\Lambda_{4}$ is the GR cosmological constant. Observe that this 'singularity' would be interpreted as a sudden singularity in the sense of [10] (generalising those in [1]]), as the scale factor $a$ remains finite but $a^{\prime}$ becomes unbounded. They have also been termed as 'type III' singularities [12] and 'big freeze' 13], see also [14]. These singularities require the violation of the energy conditions [10], but of course this refers to the energy conditions satisfied by $\varrho^{(G R)}$ and $p^{(G R)}$. Hence, this violation, or equivalently the existence of phantom or dark energy components, may be just an illusion caused by a forthcoming change of signature in the brane.

The relationship between $\varrho^{(G R)}$ and the true $\varrho$ on the brane can be deduced from (9), and is given by [3]

$$
\begin{aligned}
& \frac{\kappa_{5}^{2}}{3} \varrho=\sigma \epsilon_{1}\left[\sqrt{\frac{8 \pi G}{3} \varrho^{(G R)}+\frac{\Lambda_{4}}{3}+\tilde{\lambda}^{2}}\right. \\
& \left.-\sqrt{\frac{8 \pi G}{3} \varrho^{(G R)}+\frac{\Lambda_{4}}{3}+\lambda^{2}}\right], \\
& 8 \pi G \varrho^{(G R)}+\Lambda_{4}+3 \tilde{\lambda}^{2}=\frac{27}{4 \kappa_{5}^{4} \varrho^{2}}\left(\tilde{\lambda}^{2}-\lambda^{2}+\frac{\kappa_{5}^{4}}{9} \varrho^{2}\right)^{2} .
\end{aligned}
$$

It is interesting to see that, in this setting, the GR 'singularity' $\varrho^{(G R)} \rightarrow \infty$ is manifestly due to the vanishing of the proper energy density at the change of signature.

Let us show with an explicit example how a seemingly sudden singularity can be described with a regular signature changing brane satisfying the strong energy condition. For concreteness let us look for an equation of state of the form $p=C \varrho^{\alpha}$ with $C>0$. To restrict the possible values of $\alpha$ we need to study the behaviour $\varrho$ and $p$ near the signature change.

Let us assume that $N(\xi)$ approaches zero at the signature change located at $\xi=\xi_{f}$ as $N=\left(\xi-\xi_{f}\right)^{m} M(\xi)$ where $m$ is an odd integer and $M(\xi)$ is a regular function, positive at $\xi_{f}$. Since $\dot{a}\left(\xi_{f}\right) \neq 0$ the limit (10) coming from the Lorentzian part $\left(\xi<\xi_{f}\right)$ - reduces to $\varrho \sim\left(\xi_{f}-\xi\right)^{m / 2}$ where $\sim$ means that both terms are equivalent infinitesimals. In order to get a regular $p$ we need $m \geq 3$, so that $\dot{N}\left(\xi_{f}\right)=0$. On the other hand, the conservation law (8) leads to $p \sim\left(\xi_{f}-\xi\right)^{(m-2) / 2}$, which implies $p \sim \varrho^{(m-2) / m}$ near $\xi_{f}$. Thus, if we want to keep a power law equation of state all along $\Sigma$, we must set $p=C \varrho^{(m-2) / m}$. The conservation law (주) integrates to

$$
\varrho=C^{m / 2}\left(\bar{a}^{-6 / m}-1\right)^{m / 2},
$$

where $\bar{a} \equiv a / a_{S}$ and the integration constant has been chosen as $a_{S}=a\left(\xi_{f}\right)$ in order to enable a change of signature. Inserting this into (9) we get, after some algebra,

$$
\dot{\bar{a}}^{2}=\frac{M\left(\xi_{f}-\xi\right)^{m}}{4 \beta \bar{a}^{4}\left(1-\bar{a}^{\frac{6}{m}}\right)^{m}} F(\bar{a}),
$$

where $\beta \equiv \kappa_{5}^{4} C^{m} / 9$ and

$$
\begin{aligned}
& F(\bar{a}) \equiv\left[\bar{a}^{6}(\tilde{\lambda}-\lambda)^{2}-\beta\left(1-\bar{a}^{\frac{6}{m}}\right)^{m}\right] \\
& \times\left[\bar{a}^{6}(\tilde{\lambda}+\lambda)^{2}-\beta\left(1-\bar{a}^{\frac{6}{m}}\right)^{m}\right]-4 \beta \frac{k}{a_{S}^{2}} \bar{a}^{4}\left(1-\bar{a}^{\frac{6}{m}}\right)^{m} .
\end{aligned}
$$

Solutions of (11) can be proven to satisfy that the square roots in (2) and its tilded counterpart are real.

Eq. (11) being quadratic, it contains two branches. To choose the proper one, note that $\bar{a}\left(\xi_{f}\right)=1$ by construction and $F(1)=\left(\tilde{\lambda}^{2}-\lambda^{2}\right)^{2}>0$. Furthermore, at points of $\Sigma_{L}$ near $S$, where $\xi<\xi_{f}$, we need $\bar{a}<1$ to keep a welldefined $\varrho$. Hence $\dot{\bar{a}}>0$ near $S$ and the positive square root in (11) has to be chosen. The solution for $\bar{a}(\xi)$ depends strongly on $F(\bar{a})$, and in particular on its zeros and their order. For $k=0,1$ the existence of two simple zeroes of $F(\bar{a})$ in $\bar{a} \in(0,1)$ is ensured. Let us focus for simplicity on the case $k=1$, and let $\bar{a}_{0} \in(0,1)$ denote the zero of $F(\bar{a})$ closest to $\bar{a}=1$. We must ascertain if a solution exists extending from $\bar{a}=\bar{a}_{0}$ to $\bar{a}=1$, i.e. that for a finite $\xi_{0}, \bar{a}\left(\xi_{0}\right)=\bar{a}_{0}$ holds. Integrating (11), this amounts to showing that, for some finite $\xi_{0}$,

$$
4 \beta \int_{\bar{a}_{0}}^{1} \frac{\bar{a}^{2}\left(1-\bar{a}^{\frac{6}{m}}\right)^{m / 2}}{\sqrt{F(\bar{a})}} d \bar{a}=\int_{\xi_{0}}^{\xi_{f}} M(\xi)\left(\xi_{f}-\xi\right)^{m} d \xi,
$$

with both integrals convergent. Since $F \sim\left(\bar{a}-\bar{a}_{0}\right)$ near $\bar{a}_{0}$, the integral on the left trivially converges. The integral on the right converges for any finite $\xi_{0}$, provided $M(\xi)$ stays bounded. Thus, for a large class of positive functions $M(\xi)$ (in particular for all those bounded away from zero) a finite $\xi_{0}$ fulfilling the equality does exist.

Let us assume that one such $M(\xi)$ has been chosen. The corresponding solution $\bar{a}(\xi)$ satisfies $\dot{\bar{a}}\left(\xi_{0}\right)=0$ by construction. It is easy to check that $\ddot{\bar{a}}\left(\xi_{0}\right)>0$, which implies that the negative branch of (11) must be taken for $\xi<\xi_{0}$ (near $\xi_{0}$ ). Using a similar argument as before, the solution $\bar{a}(\xi)$ increases (as $\xi$ decreases) until reaching again $\bar{a}=1$ at some finite $\xi_{b}<\xi_{0}<\xi_{f}$. In principle, $\dot{\bar{a}}$ 


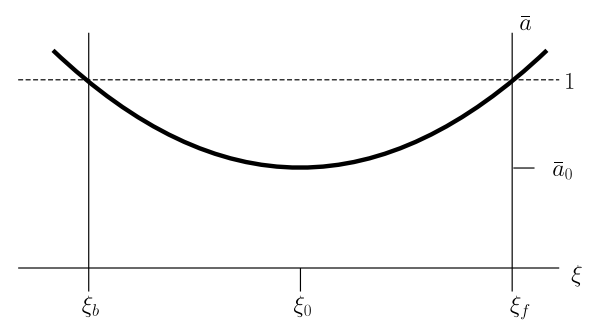

$t$
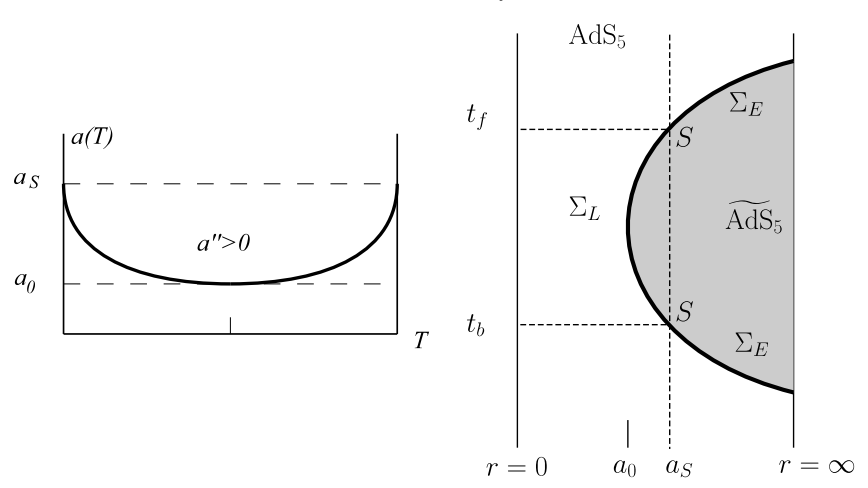

FIG. 1: The Lorentzian phase starts with a signature change at $\xi_{b}$, with a scale factor $a=a_{S}(\bar{a}=1)$ and vanishing density $\varrho$ and pressure $p$. The model contracts until it reaches the minimum scale $a_{0}$ where $\varrho$ attains its maximum, which represents a little bang (regular bounce) [15]. The model then 'bounces' to an expanding era with accelerated expansion $\left(a^{\prime \prime}>0\right)$, in which both $\varrho$ and $p$ decrease, until another change of signature occurs at $a=a_{S}$, with $\left.\varrho\right|_{S}=\left.p\right|_{S}=0$. The density and pressure remain non-negative everywhere on the Lorentzian phase. The picture on the bottom right describes the whole brane (with its Lorentzian, Euclidean and null phases) embedded in the $\mathrm{AdS}_{5}$ bulks, with $\sigma=-\epsilon_{1}=1$ and $\tilde{\lambda}<\lambda$, so that $\varrho>0$ in $\Sigma_{L}$. Observe that $\left.\varrho\right|_{\Sigma_{L}}>0$ requires $\Lambda_{5}<\widetilde{\Lambda_{5}}<0$ so that the 'eternal' $\mathrm{AdS}_{5}$ is more stable. This is very reasonable physically [1].

might diverge there, leading to a singular brane. This can be avoided if and only if $M=\left(\xi_{b}-\xi\right)^{m} P(\xi)$ for some regular function $P(\xi)$ positive in $\left[\xi_{b}, \xi_{f}\right]$. The simplest way to ensure this behaviour is to choose $M(\xi)$ even with respect to $\xi=\xi_{0}$. This implies the same even property for $\bar{a}(\xi)$. It should be stressed, however, that this is just one among many possibilities.

Let us stress that the explicit Lorentzian cosmological model, described by $a(T)$, depends on $N(\xi)$ only through its bevahiour near its zeros. The free function $P$ is irrelevant as it can always be reabsorbed with a coordinate change within the Lorentzian phase. This is already apparent in (11), from which a simple calculation gives

$$
a^{\prime \prime}=a_{S} \bar{a}^{\prime \prime}=(-1)^{m+1} \frac{a_{S}}{2} \frac{d}{d \bar{a}}\left(\frac{F(\bar{a})}{4 \beta \bar{a}^{4}\left(1-\bar{a}^{\frac{6}{m}}\right)^{m}}\right)
$$

where the dependence on $P$ has vanished.

Regarding the behaviour of the cosmic acceleration at the signature change, we need to evaluate $\bar{a}^{\prime \prime}$ in the limit $\bar{a}=1$. The calculation gives

$$
\lim _{\bar{a} \rightarrow 1} a^{\prime \prime}=\lim _{\bar{a} \rightarrow 1} \frac{2 a_{S}\left(\tilde{\lambda}^{2}-\lambda^{2}\right)^{2}}{\beta\left(1-\bar{a}^{\frac{6}{m}}\right)^{m+1}}=+\infty .
$$

Thus $a^{\prime \prime}(T)$ must be positive near the signature changes, and the seemingly 'sudden singularity' (of big-freeze type) in the future is approached while in an increasingly accelerated expansion epoch (see Fig. 1).

We have that $a^{\prime \prime}(T)$ must be positive both around $\xi_{0}$ and $\xi_{f}$, that is (i) at the beginning of the expansion epoch (when the energy density attains its maximum), which can account for an inflation era, and (ii) in the accelerated expansion epoch previous to the final 'big freeze'. On the other hand, the very particular class of models with $k=1$ and equation of state $p=C \varrho^{\alpha}$ that we have built cannot present an epoch of decelerated expansion with $a^{\prime \prime}(T)<0$-in order to stop the initial inflation-, as it can be proven by using (12). However, due to the large freedom in the equation of state it seems likely that many models will exist with $a^{\prime \prime} \leq 0$ for some period after the initial inflation epoch.

We thank L. Fernández-Jambrina and R. Lazkoz for their suggestions, and also M. Bouhmadi-López for her comments. MM was supported by the projects FIS200605319 of the Spanish MEC and SA010CO5 of the Junta de Castilla y León. JMMS thanks support under grants FIS2004-01626 (MEC) and GIU06/37 of the University of the Basque Country (UPV). RV is funded by the Basque Government Ref. BFI05.335 and thanks support from project GIU06/37 (UPV).

[1] M. Mars, J.M.M. Senovilla, R. Vera 2001 Phys. Rev. Lett. 864219

[2] G.W. Gibbons, A. Ishibashi, 2004 Class. Quantum Grav. 212919

[3] M. Mars, J.M.M. Senovilla, R. Vera 2007 Phys. Rev. D 76044029

[4] N. Arkani-Hamed, S. Dimopoulos, G. Dvali 1998 Phys. Lett. B 429 263; L. Randall, R. Sundrum, 1999 Phys. Rev. Lett. 83 3370; - 1999 Phys. Rev. Lett. 834690

[5] R. Maartens (2004) "Brane-World Gravity", Living Rev. Relativity 7, 3. URL (cited on 29 September 2007): http://www.livingreviews.org/lrr-2004-7

[6] Y. Shtanov, V. Sahni 2002 Class. Quantum Grav. 19 L101

[7] R. Vera 2002 Class. Quantum Grav. 195249

[8] M. Mars, J.M.M. Senovilla 1993 Class. Quantum Grav. 101865

[9] F. Fayos, J.M.M. Senovilla, R. Torres 1996 Phys. Rev. D 544862

[10] C. Cattoën and M. Visser 2005 Class. Quantum Grav. 22 4913

[11] J.D. Barrow 2004 Class. Quantum Grav. 21 L79; ibid 5619

[12] S. Nojiri, S.D. Odintsov and S. Tsujikawa 2005 Phys. Rev. D $\mathbf{7 1} 063004$ 
[13] M. Bouhmadi-López, P. F. González-Díaz, P. MartínMoruno arXiv:gr-qc/0612135v1

[14] L. Fernández-Jambrina and R. Lazkoz, 2006 Phys. Rev.
D $\mathbf{7 4 0 6 4 0 3 0}$

[15] J.M.M. Senovilla, 1998 Gen. Rel. Grav. 30701 (sect. 7.7) 